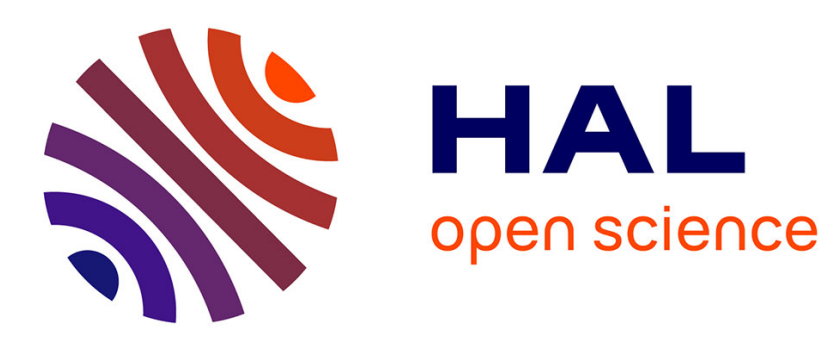

\title{
Molecular orientation and anchoring energy at the nematic-isotropic interface of $7 \mathrm{CB}$
}

\author{
S. Faetti, V. Palleschi
}

\section{To cite this version:}

S. Faetti, V. Palleschi. Molecular orientation and anchoring energy at the nematic-isotropic interface of 7CB. Journal de Physique Lettres, 1984, 45 (7), pp.313-318. 10.1051/jphyslet:01984004507031300 . jpa-00232349

\section{HAL Id: jpa-00232349 https://hal.science/jpa-00232349}

Submitted on 1 Jan 1984

HAL is a multi-disciplinary open access archive for the deposit and dissemination of scientific research documents, whether they are published or not. The documents may come from teaching and research institutions in France or abroad, or from public or private research centers.
L'archive ouverte pluridisciplinaire HAL, est destinée au dépôt et à la diffusion de documents scientifiques de niveau recherche, publiés ou non, émanant des établissements d'enseignement et de recherche français ou étrangers, des laboratoires publics ou privés. 
Classification

Physics Abstracts

$61.30-68.10-64.70$

\title{
Molecular orientation and anchoring energy at the nematic-isotropic interface of $7 \mathrm{CB}\left({ }^{*}\right)$
}

\author{
S. Faetti (**) and V. Palleschi \\ Dipartimento di Fisica, Piazza Torricelli, 2, 56100 Pisa, Italy
}

(Reçu le 20 décembre 1983, acceptéle 7 février 1984)

\begin{abstract}
Résumé. - Nous étudions par réflectométrie optique les propriétés de l'interface nématique-isotrope du cristal liquide 7CB. Nous mesurons l'épaisseur de l'interface, l'angle polaire du directeur à l'interface et son énergie d'ancrage.

Abstract. - The properties of the nematic-isotropic interface of the nematic liquid crystal 7CB are studied by optical reflectometry. It is found that at the interface the director is tilted with respect to the vertical axis. Measurements are reported of the tilt angle $\theta_{t}$, of the thickness of the interface, and of the anchoring energy.
\end{abstract}

\section{Introduction.}

In recent years there has been a growing interest in the orientational properties of molecules at the interface between a nematic liquid crystal (LC) and another non-nematic medium [1-12]. In particular, the free surface of some nematic liquid crystals [3-10] and the nematic-isotropic interface of MBBA have been investigated [11, 12]. In this paper we study by optical reflectometry the properties of the nematic-isotropic interface of the nematic liquid crystal 4-cyano-4'-n-heptylbiphenyl (7CB) [13]. We measure both the thickness of the interface and the director orientation at the interface. Moreover, we also give the first experimental measurement of the anchoring energy of the director at a nematic-isotropic interface.

\section{Experimental apparatus.}

The nematic sample we use is a commercial 7CB produced by $\mathrm{BDH}$, which shows the proper clearing temperature given in the literature [13]. The geometry of the experimental cell containing the nematic sample is analogous to the one already used by D. Langevin and M. A. Bouchiat [11] in order to study the nematic-isotropic interface of MBBA. The sample is bounded by two horizontal glass plates. These plates are in contact with two copper plates maintained at two different temperatures in order to generate a temperature gradient parallel to the gravity field. The upper region of the $\mathrm{LC}$ is in the isotropic phase, while the lower region is in the nematic phase. A small

$\left(^{*}\right)$ Research supported in part by MPI and in part by CNR through GNSM, unit of Pisa.

$\left({ }^{* *}\right)$ Gruppo Nazionale di Struttura della Materia del CNR. 
hole (diameter $3 \mathrm{~mm}$ ) is opened in the upper copper plate in order to allow an incident laser beam to pass through. The temperature of both the plates is held fixed to within $\pm 0.05^{\circ} \mathrm{C}$. A plexiglas box ensures the thermal insulation of the lateral walls of the cell from outside. The sample lies between the polar expansions of an electromagnet which generates a magnetic field variable between $0.2 \mathrm{kG}$ and $7.6 \mathrm{kG}$.

The experimental apparatus used for measuring the optical reflectivity coefficients at the nematic-isotropic interface is schematically shown in figure 1 . The glass plate $\mathrm{G}$ splits the He-Ne laser beam $(\lambda=6328 \AA)$ into two beams. One is collected by the photodiode $\mathrm{PH}_{1}$ that measures its intensity $I_{0}$, the other is polarized at $45^{\circ}$ with respect to the magnetic field by the polarizer $\mathrm{P}$ and impinges with an incidence angle of $0.9^{\circ}$ on the nematic-isotropic interface. The reflected beam passes through a Wollaston prism which splits the optical beams polarized in a direction parallel or orthogonal to the magnetic field. The intensities $I_{\|}$and $I_{\perp}$ of the two beams are measured by the photodiodes $\mathrm{PH}_{2}$ and $\mathrm{PH}_{3}$, respectively. Two analogic dividers allow us to obtain the ratios $I_{\|} / I_{0}$ and $I_{\perp} / I_{0}$ with an accuracy better than $0.2 \%$. In order to calibrate the response of the whole apparatus, we have measured the ratios $I_{\|} / I_{0}$ and $I_{\perp} / I_{0}$ when the nematic-isotropic interface was replaced by a glass plate of known refractive indices.

\section{Principle of measurements.}

The principle underlying our measurements has been already described by D. Langevin and M. A. Bouchiat [11] and by P. Chiarelli et al. [5]; thus we only summarize here the main ideas. In order to measure the surface angle $\theta_{t}$, the director at the nematic-isotropic interface should be aligned along a well defined direction. This condition is satisfied by applying a horizontal magnetic field. In this case, the polar angle of the director in the bulk is a function of the $z$-coordinate that ranges from the surface value $\theta_{0}=\theta(H)>\theta_{\mathrm{t}}$ to the bulk value $\theta(-\infty)=90^{\circ}$. The thickness of the distorted layer is of the order of a few magnetic coherence lengths $\xi_{H}$ [14]. If the nematicisotropic interface is not sharp, but has a diffuse profile with " effective thickness " $L$, the reflectivity coefficients for the extraordinary and ordinary waves, will be given in principle by :

$$
R_{\|}=R_{\|}\left(n_{\|}, n_{\perp}, n_{\text {iso }}, \theta_{0}, \theta_{\mathrm{i}}, \xi_{H}, L\right)
$$

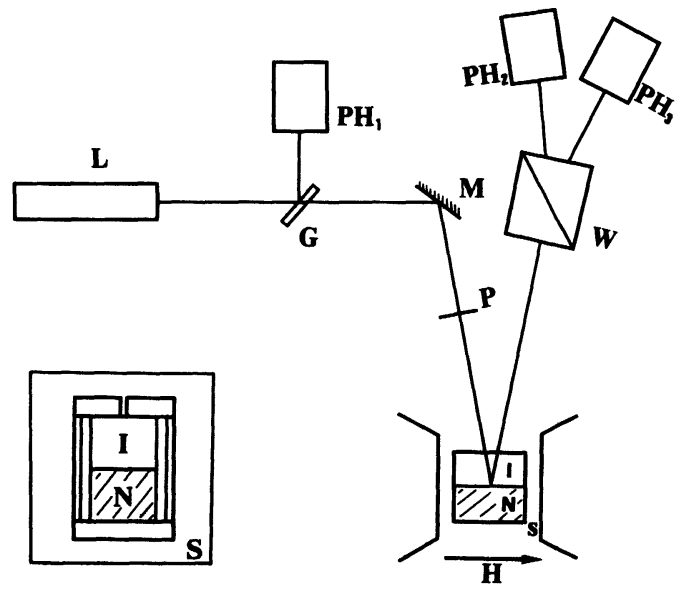

Fig. 1. - Experimental apparatus : $\mathrm{L}=$ laser source; $\mathrm{PH}_{1}, \mathrm{PH}_{2}, \mathrm{PH}_{3}=$ photodiodes $; \mathrm{G}=$ glass plate; $\mathbf{M}=$ mirror $; \mathbf{P}=$ polarizer $; \mathbf{W}=$ Wollaston prism; $\mathbf{S}=$ sample $; \mathbf{I}=$ isotropic phase $; \mathbf{N}=$ nematic phase; $H=$ magnetic field. The incidence angle of the laser beam is $0.9^{\circ}$. A schematic cross section of the thermostatic box is shown in the insert. 
and

$$
R_{\perp}=R_{\perp}\left(n_{\perp}, n_{\text {iso }}, \theta_{\mathrm{i}}, L\right)
$$

where $n_{\|}$and $n_{\perp}$ are the extraordinary and ordinary indices, respectively, of the nematic LC at the clearing temperature $T_{\mathrm{c}}, n_{\mathrm{iso}}$ is the index of the isotropic phase at $T=T_{\mathrm{c}}$ and $\theta_{\mathrm{i}}$ is the incidence angle. In general, the theoretical values of $R_{\|}$and $R_{\perp}$ cannot be given in analytic form, but can be deduced by numerical integration of the Maxwell equations [15]. In order to account for the presence of a diffuse interface $(L \neq 0)$, we must assume a well-defined expression for the dependence of the refractive indices $n_{\perp}$ and $n_{\|}$on the $z$ vertical coordinate near the interface. We choose $[16,17]$ :

$$
n_{j}(z)=\frac{1}{2}\left[n_{j}+n_{\text {iso }}+\left(n_{\text {iso }}-n_{j}\right) \tanh \left(\frac{2 z}{L}\right)\right]
$$

where $j=\mathrm{i}, 2$ corresponds to $\perp$ and $/ /$, respectively. We have easily verified that equation (3) agrees with the $z$-dependence of the scalar order parameter $S$ as obtained by minimizing the Landau-De Gennes surface free energy $\gamma[14]$ with respect to $S$; where :

$$
\gamma=\int_{-\infty}^{+\infty} \mathrm{d} z\left[\Phi_{0}+\frac{3}{4} A S^{2}-\frac{1}{3} B S^{3}+\frac{1}{4} C S^{4}+\frac{3}{4} A \xi^{2}\left(\frac{\mathrm{d} S}{\mathrm{~d} z}\right)^{2}\right] .
$$

In this case $L$ is related to the correlation length $\xi$ by the simple expression : $L=4 \xi$. Note that the $L$ parameter can also account for the effects due to thermal deflections of the nematic-isotropic interface, as has been recently pointed out by J. Meunier and D. Langevin [16]. Therefore, we will include these effects too in the " effective " thickness $L$. Since the refractive indices $n_{\perp}, n_{\|}$ and $n_{\text {iso }}$ of 7CB are known [18], integration of the Maxwell equations allows us to obtain the theoretical dependence of $R_{\perp}$ on $L$ and of $R_{\|}$on $L$ and $\theta_{0}$.

The experimental analysis proceeds in two successive steps : first, from the measured value of $R_{\perp}$ we deduce the effective thickness $L$, then from the value of the ratio $R_{\|} / R_{\perp}$ we deduce $\theta_{0}=$ $\theta(H)$ for different values of the applied magnetic field.

\section{Experimental results.}

Some remarks are now appropriate before discussing the experimental results. First of all, we notice that the experimental values of $R_{\perp}$ and $R_{\|}$must be corrected in order to account for the losses due to the upper glass plate of the cell and to the light scattering from the upper isotropic LC. In order to make this correction, we have changed the temperature $T_{2}$ of the bottom plate without changing the vertical temperature gradient. If the temperature of this plate is greater than $T_{c}$, the whole $\mathrm{LC}$ is in the isotropic phase and the intensity of the laser beam reflected by the upper surface of the bottom glass plate can be measured. In these conditions we decrease the temperature $T_{2}$ very slowly $\left(\Delta T / \Delta t=0.01{ }^{\circ} \mathrm{C} / \mathrm{min}\right)$ and we record the intensity of the reflected beam with time. This intensity shows a small decrease due to increasing light scattering from the isotropic phase of the LC [19]. When the temperature of the bottom plate approaches $T_{c}$, the adjacent LC makes a transition to the nematic phase. In these conditions the reflected intensity shows a very sharp decrease due to the light scattering by the nematic phase (Fig. 2). The ratio between the reflected intensity just before $T_{\mathrm{c}}$ and the intensity reflected by an identical reference glass plate allows us to measure the losses due both to the upper glass plate of the cell $(\sim 13 \%)$ and to the isotropic nematic LC $(\sim 5 \%)$. In our experiment these losses are found to be $\Delta I / I=$ $0.18 \pm 0.04$. We have also verified that both the Faraday effect of the upper glass window and the critical magnetic birefringence of the isotropic phase of $7 \mathrm{CB}$ do not affect appreciably the measured value of $R_{\|} / R_{\perp}$. In fact, the intensities $I_{\perp}$ and $I_{\|}$of the two beams reflected from the bottom 


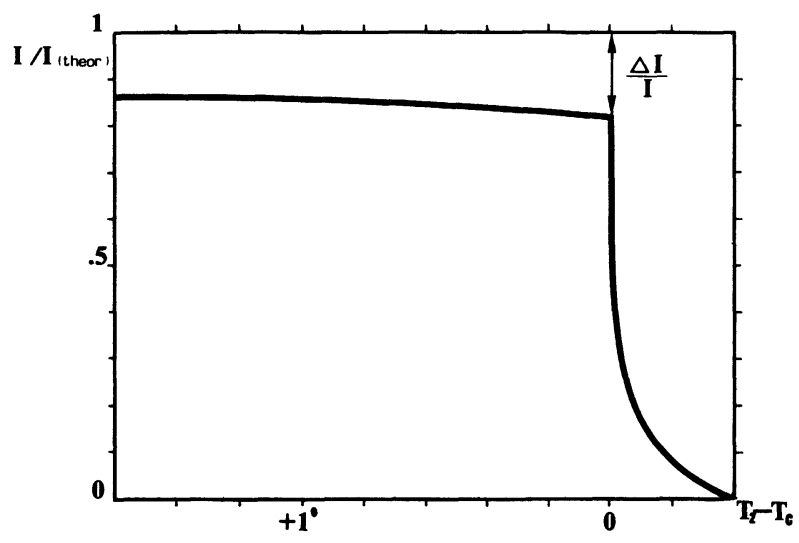

Fig. 2. - Intensity of the reflected beam from the bottom glass surface of the nematic cell as a function of the difference between the temperature $T_{2}$ of this surface and the clearing temperature $T_{\mathrm{c}}$. The vertical temperature gradient is $10^{\circ} \mathrm{C} / \mathrm{cm}$. The measured intensity is divided by that reflected from an identical glass plate in absence of losses $\left(I_{\text {theor }}\right)$.

glass plate do not depend on the intensity of the magnetic field within our experimental accuracy $(\sim 5 \%)$. Finally, the experimental results on $7 \mathrm{CB}$ are not sensitive to the intensity of the temperature gradient in the range from $1{ }^{\circ} \mathrm{C} / \mathrm{cm}$ to $15{ }^{\circ} \mathrm{C} / \mathrm{cm}$. This observation indicates that thermal instabilities do not influence our measurements.

$R_{\perp}$ and $R_{\|}$versus the applied magnetic field are shown in figure 3. Let us notice that in principle, the magnetic field can affect the order parameter $S$ and thus, the extraordinary and ordinary indices of the nematic sample. However in our experiment we find $R_{\perp}$ is independent of $H$; therefore this effect does not affect our experimental measurements of $\theta_{0}(H)$. The experimental points for high magnetic fields $(H>1.5 \mathrm{kG})$ are more reproducible and are completely independent of the incidence point at the nematic-isotropic interface. On the contrary, the experimental points for $H<1.5 \mathrm{kG}$ are sensitive to the incidence point. The latter dependence is due to an incomplete alignment of the director along the $x$-horizontal axis parallel to the magnetic field. Therefore, the angle $\theta_{t}$ is determined to a larger accuracy by extrapolating to $H=0$ only the experimental results obtained at high field intensities (continuous lines in Fig. 3). From the $R_{\perp}$ data and by using $n_{\|}=1.6385, n_{\perp}=1.5335$ and $n_{\text {iso }}=1.564$ as experimental values of the refractive indices of 7CB deduced by reference [18] and extrapolated to the wavelength $\lambda=6328 \AA$, we find :

$$
L=500 \pm 240 \AA \text {. }
$$

Notice that the high experimental uncertainty $( \pm 240 \AA)$ is mostly due to the uncertainty of the refractive indices reported in the literature. In fact, by comparing experimental values of $n_{\perp}$ and $n_{\|}$of reference [18] with those of reference [20] we find differences of the order of $\Delta n= \pm$ 0.002 , which gives a maximum relative error in the expected reflectivity coefficient $R_{\perp}$ of the order of $25 \%$. Minor source of experimental errors are :

1) The uncertainty of the alignment of the principal axes of the Wollaston prism with respect to the magnetic field $(\Delta R / R \sim 4 \%)$.

2) The uncertainty due to the spurious signal generated by the light scattered from the glass window and impinging on the photodetector $(\Delta R / R \sim 5 \%)$.

3) The uncertainty on the corrections for the losses from the glass window and the isotropic $\mathrm{LC}(\Delta R / R \simeq 3 \%)$. 


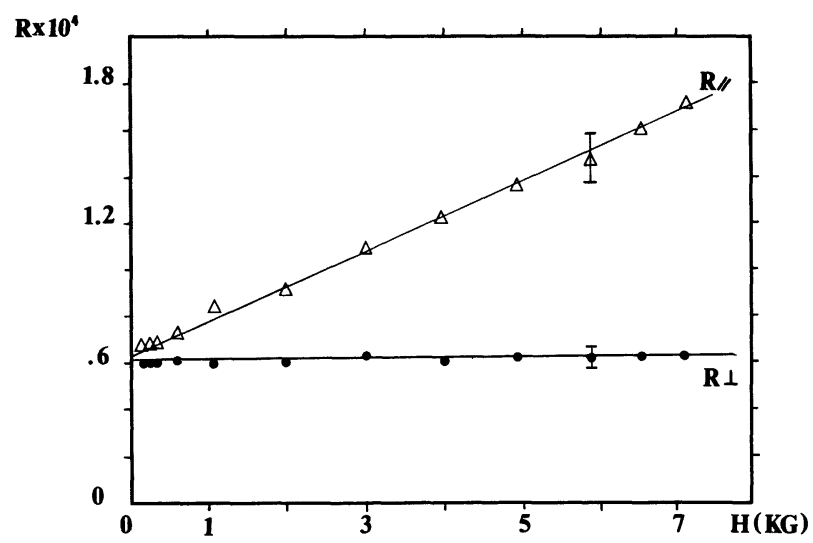

Fig. 3. - Orthogonal $(\bullet)$ and parallel $(\Delta)$ intensity reflectivity coefficients at the nematic-isotropic interface of $7 \mathrm{CB}$ as a function of the magnetic field. Each point corresponds to the average value of two different sets of measurements.

Errors due to the residual spurious anisotropy of the glass window are negligible. In order to find the polar angle $\theta_{t}$ at the interface we extrapolate to $H=0$ the experimental values of $R_{\|} / R_{\perp}$ obtained in the range $H>1.5 \mathrm{kG}$. By comparing the experimental value $\lim _{H \rightarrow 0} R_{\|} / R_{\perp}$ with the theoretical one for different values of the polar angle $\theta_{\mathrm{t}}$ one obtains :

$$
\theta_{\mathrm{t}}=52 \pm 6^{\circ}
$$

In this case as well, the experimental uncertainty is mostly due to the uncertainty of the known values of the refractive indices. In fact the reproducibility between different measurements is better than $\pm 1.5^{\circ}$. Notice that the experimental result $R_{\|}(0) \sim R_{\perp}(0)$ is also consistent with an homeotropic alignment of the director at the interface. However if $\theta_{t}=0$ the ratio $R_{\|} / R_{\perp}$ should be a decreasing function of the magnetic field. This remark allows us to reject this possibility.

The anchoring energy coefficient of the director at the nematic-isotropic interface can be deduced by measuring the coefficient of linear dependence between $\theta_{0}$ and $H$.

We find :

$$
\frac{\Delta \theta}{\Delta H}=(3.8 \pm 0.5) \cdot 10^{-5} \mathrm{rad} / \mathrm{G}
$$

By using the Frank-Ericksen elastic.theory [21] in the approximation of isotropic elastic cons$\operatorname{tants}\left(K_{11}=K_{33}=\bar{K}\right)$ and by assuming for the anchoring energy near the equilibrium point the simple analytical form :

$$
W_{\mathrm{a}}=W\left(\theta_{0}-\theta_{\mathrm{t}}\right)^{2}
$$

one obtains easily the theoretical dependence (for $\theta_{0}-\theta_{t} \ll 1$ ) :

$$
W=\frac{\sqrt{\bar{K} \chi_{\alpha}} \cos \theta_{\mathrm{t}} \Delta H}{2 \Delta \theta}
$$

where $\bar{K}$ is the average elastic constant and $\chi_{\alpha}$ is the anisotropy of the magnetic susceptibility. By 
using the values of $\bar{K}$ and $\chi_{\alpha}$ measured by P. P. Karat and N. V. Madhusudana [22], we obtain for the anchoring energy coefficient the value :

$$
W=(1.8 \pm 0.4) \cdot 10^{-3} \mathrm{erg} /\left(\mathrm{cm}^{2} \mathrm{rad}^{2}\right)
$$

which is very small.

\section{References}

[1] Croxton, C. A., Chandrassekar, S., Pramana Suppl. 1 (1975) 237.

[2] Cognard, J., Mol. Cryst. Liq. Cryst. (Suppl. 1)(1982) 1.

[3] Bouchiat, M. A. and Langevin-Cruchon, D., Phys. Lett. 34 (1971) 331.

[4] Proust, J. E. and Ter-Minassian-Saraga, L., Colloid Polym. Sci. 259 (1977) 1133.

[5] Chiarelli, P., Faetti, S. and Fronzoni, L., J. Physique 44 (1983) 1061.

[6] Parsons, J. D., J. Physique 37 (1976) 1187.

[7] Parsons, J. D., Phys. Rev. Lett. 41 (1978) 877.

[8] Mada, H., Mol. Cryst. Liq. Cryst. 53 (1979) 127.

[9] Mada, H., Mol. Cryst. Liq. Cryst. 51 (1979) 43.

[10] Horn, R. G., Israelachvili, J. N. and Perez, E., J. Physique 42 (1981) 39.

[11] Langevin-Cruchon, D. and Bouchiat, M. A., Mol. Cryst. Liq. Cryst. 22 (1973) 317.

[12] Vilanove, R., Guyon, E., Mitescu, C. et Pieranski, P., J. Physique 35 (1974) 153.

[13] Gray, G. W., J. Physique Colloq. 36 (1975) C1-337.

[14] De Gennes, P. G., The Physics of Liquid Cristals (Clarendon Press Oxford) 1974.

[15] Berremann, D. W., J. Opt. Soc. Am. 62 (1972) 502.

[16] Meunier, J. and Langevin, D., J. Physique Lett. 43 (1982) L-185.

[17] Huang, J. S. and Webb, W. W., J. Chem. Phys. 50 (1969) 3677.

[18] Dunmur, D. A., Manterfield, M. R., Miller, W. H. and Dunleavy, J. K., Mol. Cryst. Liq. Cryst. 45 (1978) 127.

[19] Coles, H. J. and Strazielle, C., Mol. Cryst. Liq. Cryst. 55 (1979) 237.

[20] Karat, P. P. and Madhusudana, N. V., Mol. Cryst. Liq. Cryst. 36 (1976) 51.

[21] Frank, F. C., Discuss. Faraday Soc. 25 (1958) 19.

[22] Karat, P. P. and Madhusudana, N. V., Mol. Cryst. Liq. Cryst. 40 (1977) 239. 